\title{
Platelet-stored angiogenesis factors: Clinical monitoring is prone to artifacts
}

\author{
Patrick Starlinger, Lejla Alidzanovic, Dominic Schauer, Philipp Brugger, Silvia Sommerfeldt, \\ Irene Kuehrer, Sebastian F. Schoppmann, Michael Gnant and Christine Brostjan* \\ Department of Surgery, General Hospital, Medical University of Vienna, Vienna, Austria
}

\begin{abstract}
Background: The analysis of angiogenesis factors in the blood of tumor patients has given diverse results on their prognostic or predictive value. Since mediators of angiogenesis are stored in platelets, their measurement in plasma is sensitive to inadvertent platelet activation during blood processing.

Methods: Variants of blood withdrawal and plasma preparation were evaluated by ELISA for the detection of TSP-1, PF-4, VEGF and PD-ECGF. A total of 22 pancreatic cancer patients and 29 healthy volunteers were evaluated.

Results: Plasma preparation with the anticoagulant mix of citrate, theophylline, adenosine, dipyridamole (CTAD) and immediate blood processing at $4{ }^{\circ} \mathrm{C}$ was required for reproducible measurements of TSP-1, PF-4 and VEGF. Blood collection by venflon or inadvertent hemolysis during blood withdrawal caused significantly elevated TSP-1 and PF4 values. When optimized plasma preparation was applied, a significant increase of TSP-1 and VEGF in cancer patients was detected $(\mathrm{P}=0.006 ; \mathrm{P}<0.001)$. Conclusion: The reliable plasma analysis of circulating platelet-stored angiogenesis factors requires preparation with CTAD at $4^{\circ} \mathrm{C}$ and blood collection by butterfly needle. Suboptimal procedures of plasma preparation are commonly applied in clinical monitoring of angiogenesis parameters which may account for the differences in reported plasma values and may have masked their predictive or prognostic marker potential.
\end{abstract}

Keywords: Angiogenesis factors, cancer, plasma, platelet derived endothelial cell growth factor, platelet factor 4, thrombospondin 1 , vascular endothelial growth factor

\section{Introduction}

Angiogenesis is the physiological process of capillary sprouting and remodeling of an established network of blood vessels, and plays a central role in embryogenesis, the female menstruation cycle, as well as wound healing [1]. Moreover, angiogenesis is increasingly recognized to be involved in diseases such as proliferative retinopathy, rheumatoid arthritis, psoriasis and all types of malignancies [2]. The neovascularization process requires a tightly regulated balance of pro- and anti-angiogenic factors, among those the

\footnotetext{
* Corresponding author: Christine Brostjan, Department of Surgery, Medical University of Vienna, Anna Spiegel Research Laboratories, General Hospital AKH 25.05.002, Waehringer Guertel 18-20, A-1090 Vienna, Austria. Tel.: +43 140400 73514; Fax: +43 1 40400 6782; E-mail: christine.brostjan@meduniwien.ac.at.
}

highly potent and specific vascular endothelial growth factor (VEGF). VEGF was shown to promote angiogenesis by inducing endothelial cell proliferation, migration and tube formation [3]. In the tumor setting, VEGF is produced by tumor or stroma cells and is highly abundant in platelet $\alpha$-granules [4]. Inhibition of VEGF was found to reduce tumor growth and has thus been introduced to clinical application [5]. Furthermore, circulating VEGF values have shown prognostic or predictive potential for various types of cancer $[6,8]$.

In addition to VEGF a variety of other platelet-stored angiogenesis factors potently affect physiological and pathological vessel growth. For example, the major anti-angiogenic factors thrombospondin 1 (TSP-1) and platelet factor 4 (PF-4) are also abundantly stored in $\alpha$ granules and released upon platelet activation. While circulating PF-4 is rapidly bound to endothelial cells, soluble TSP-1 is more stable in circulation [9]. Apart 
from their essential role in hemostasis and thrombosis $[10,12]$, both factors have been shown to inhibit angiogenesis and suppress tumor growth [13,14]. Furthermore, metastatic tumor spread, tumor cell adhesion and invasion were shown to be regulated by TSP- 1 [15, 16]. Interestingly, reports on the marker potential of TSP-1 and PF-4 in the blood of cancer patients have been highly controversial $[15,17,18]$.

In contrast to VEGF, TSP-1 and PF-4 which are stored in platelet $\alpha$-granules, other regulators of neovascularization such as the pro-angiogenic platelet derived endothelial cell growth factor (PD-ECGF) are found in the cytoplasm of platelets [19] and are therefore not secreted upon platelet degranulation. PDECGF is released by platelets, tumor or stroma cells upon cell lysis and promotes survival and chemotaxis of endothelial cells. Studies on the marker potential of PD-ECGF are limited, but PD-ECGF has been suggested to be associated with tumor progression and metastasis [20].

Based on the central role of VEGF in the neovascularization process, monitoring of angiogenic blood parameters has predominantly focused on VEGF analysis while comparably little attention has been given to the detection of other angiogenesis factors. Thus, a major debate was raised in the late 1990s concerning the optimal blood specimen for circulating VEGF measurements. A variety of plasma and serum preparations were evaluated to define the optimized procedure for VEGF detection in patient blood [21,22]. Since serum preparation leads to platelet activation, serum values mainly reflect platelet-released VEGF. It was of interest to note that VEGF stored in platelets correlated with tumor burden presumably due to scavenging of tumor-derived VEGF [23]. Thus, serum measurements seemed to be applicable to VEGF evaluation. However, in cancer patients platelet counts may be affected by myelosuppressive chemotherapy. Additional plasma measurements are therefore advisable when monitoring patients under therapy $[24,25]$. For plasma analysis substantial differences concerning platelet activation by different preparation variants were recorded; it was subsequently proposed to use the anticoagulant mix of citrate, theophylline, adenosine, dipyridamole (CTAD) and to process blood samples at $4^{\circ} \mathrm{C}$ to avoid platelet degranulation when determining plasma VEGF levels [21].

Despite the thorough evaluation of blood processing methods for reliable VEGF detection conducted 10 years ago, clinical VEGF monitoring has largely continued with suboptimal procedures and processing methods have not been evaluated for the majority of other angiogenesis factors. In contrast, the monitoring of angiogenesis parameters has gained importance over the last years, since anti-angiogenic agents have been introduced into cancer treatment regimens. In this context, circulating angiogenesis factors have been investigated for their prognostic and predictive potential but have mostly shown disappointing outcome [8]. As angiogenesis parameters beside VEGF may be crucially affected by blood preparation and analysis methods, the results may be hampered by suboptimal procedures. We therefore set out to evaluate the impact of blood processing variants and define an optimized procedure suitable to reliably measure angiogenesis factors, with particular emphasis on platelet-stored proteins such as VEGF, TSP-1, PD-ECGF and PF-4.

\section{Materials and methods}

\subsection{Study collective of healthy volunteers and cancer patients}

Twenty-nine healthy volunteers were included in the study and divided into three groups: 3 subjects were recruited for the comparison of serum and plasma preparation variants; 3 volunteers contributed to the evaluation of blood storage time; 10 were enrolled to evaluate the impact of blood withdrawal procedures and 13 volunteers with a median age of 59 years (ranging from 41 to 73 years) served as age-matched healthy control for the collective of pancreatic carcinoma patients.

Blood samples of 22 patients with locally advanced, non-metastatic pancreatic carcinoma were drawn to monitor angiogenic factors before and during therapy. Nineteen patients (median age of 63, ranging from 43 to 80 years) received gemcitabine at $1000 \mathrm{mg} / \mathrm{m}^{2}$ on days 1,8 , and 15 of four consecutive 4 -week cycles with or without the bi-weekly addition of bevacizum$\mathrm{ab}$ at $5 \mathrm{mg} / \mathrm{kg}$. Blood samples were collected every two weeks, starting from the first time of chemotherapy administration.

The analysis of blood samples was approved by the Institutional Ethics Committee; all patients and healthy volunteers gave written informed consent. Furthermore this study conforms with The Code of Ethics of the World Medical Association (Declaration of Helsinki) as printed in the British Medical Journal (18th of July 1964). 


\subsection{Plasma and serum preparation}

For optimized plasma preparation, blood was drawn into chilled CTAD-tubes, immediately put on ice and further processed within $30 \mathrm{~min}$. After an initial centrifugation step at $1000 \mathrm{x} \mathrm{g}$ and $4^{\circ} \mathrm{C}$ for $10 \mathrm{~min}$, the plasma supernatant was subjected to further centrifugation at $10000 \mathrm{x} \mathrm{g}$ and $4{ }^{\circ} \mathrm{C}$ for $10 \mathrm{~min}$ (to remove "contaminating" platelets). The supernatant was stored in aliquots at $-80^{\circ} \mathrm{C}$ to avoid repeated cycles of freezing and thawing before analysis.

To evaluate the effect of different blood sampling methods on the detection of platelet-stored angiogenesis factors, blood was collected in EDTA, CIT (citrate), or CTAD tubes. All samples were further processed as described for the optimized plasma preparation method at $4{ }^{\circ} \mathrm{C}$. Additionally, blood processing with the various anticoagulants was also performed at room temperature. Serum samples were retrieved by blood collection without the addition of anticoagulants and by centrifugation (at $1000 \mathrm{x} \mathrm{g}$ and RT for $10 \mathrm{~min}$ ) $30 \mathrm{~min}$ or 2 hours after collection.

Furthermore, to determine if differences in blood withdrawal methods can affect measurements of circulating angiogenic factors, Venflon ${ }^{\mathrm{TM}}$ (18 G: 1.270 mm needle diameter) and Vacutainer ${ }^{\circledR}$ butterfly $(21 \mathrm{G}$ : $0.813 \mathrm{~mm}$ needle diameter) collection systems by Becton Dickinson (Franklin Lakes, NJ) were compared for 10 healthy individuals by concomitant blood withdrawal with both variants.

\subsection{Determining concentrations of angiogenesis factors}

Plasma or serum samples were analyzed by ELISA to determine the concentration of circulating angiogenesis factors. Commercially available ELISA tests were applied for VEGF and TSP-1 (Quantikine; R\&D Systems, Minneapolis, MN) as well as platelet factor 4 (Asserachrom PF4; Diagnostica Stago, Asnières, France) according to manufacturers' instructions. A comparable "sandwich" ELISA system for PD-ECGF (detection range: $1-100 \mathrm{ng} / \mathrm{mL}$ ) has previously been reported by us [26,27] and is based on the following antibodies: gelatin-free goat polyclonal $\alpha$-human PDECGF antiserum (sc-9523; Santa Cruz Biotechnology, Inc., Santa Cruz, CA) diluted to $5 \mu \mathrm{g} / \mathrm{mL}$ for coating, and murine monoclonal $\mathrm{IgG}_{1}$ clone PGF.44C diluted to $500 \mathrm{ng} / \mathrm{mL}$ for detection (Lab Vision Corp., Thermo Fisher Scientific Inc., Fremont, CA). The detection antibody was biotinylated using the FluoRe- porter Mini-Biotin-XX Protein Labeling kit (Molecular Probes, Invitrogen Corp., Carlsbad, CA) according to manufacturer's instructions and was further complexed with a conjugate of streptavidin-horseradish peroxidase (Pierce Biotechnology Inc., Rockford, IL). Plasma samples were measured undiluted at $50 \mu \mathrm{L}$ per well and compared with a standard dilution series of human recombinant PD-ECGF (R\&D Systems).

\subsection{Quantitative analysis of plasma preparation variants applied in reported studies on angiogenesis parameters}

To evaluate the frequency of anticoagulants used to determine plasma values of angiogenesis factors in reported studies, the PubMed database was searched for articles published within the last 2 years. The following terms were used to identify relevant studies: (vascular endothelial growth factor OR VEGF) AND plasma; (thrombospondin-1 OR TSP-1) AND plasma; (platelet factor 4 OR PF-4) AND plasma; (PD-ECGF OR platelet derived endothelial cell growth factor OR thymidine phosphorylase) AND plasma. With respect to VEGF the first 100 of 530 retrieved articles were analyzed as a representative fraction of reported studies.

\subsection{Statistical analysis}

Statistical analyses were carried out with SPSS 17.0.1 Software (SPSS, Inc., Chicago, IL) and were based on non-parametric tests (Spearman, Mann Whitney $\mathrm{U}$, and Wilcoxon test).

\section{Results}

\subsection{Blood measurements of angiogenesis factors stored in platelet granules are highly sensitive to blood processing variants}

To evaluate the impact of blood processing methods on detectable plasma values of various platelet-stored angiogenic factors, the anticoagulants CTAD, citrate and EDTA were compared with processing at $4{ }^{\circ} \mathrm{C}$ or room temperature. Additionally, serum was prepared in the absence of anticoagulants at room temperature. Coagulation times of $30 \mathrm{~min}$ and $2 \mathrm{~h}$ were applied.

VEGF, TSP-1, PF-4 and PD-ECGF fluctuations in different plasma and serum preparations were analyzed by ELISA in blood samples of three healthy volunteers (1 female, 2 male) and three pancreatic cancer patients 

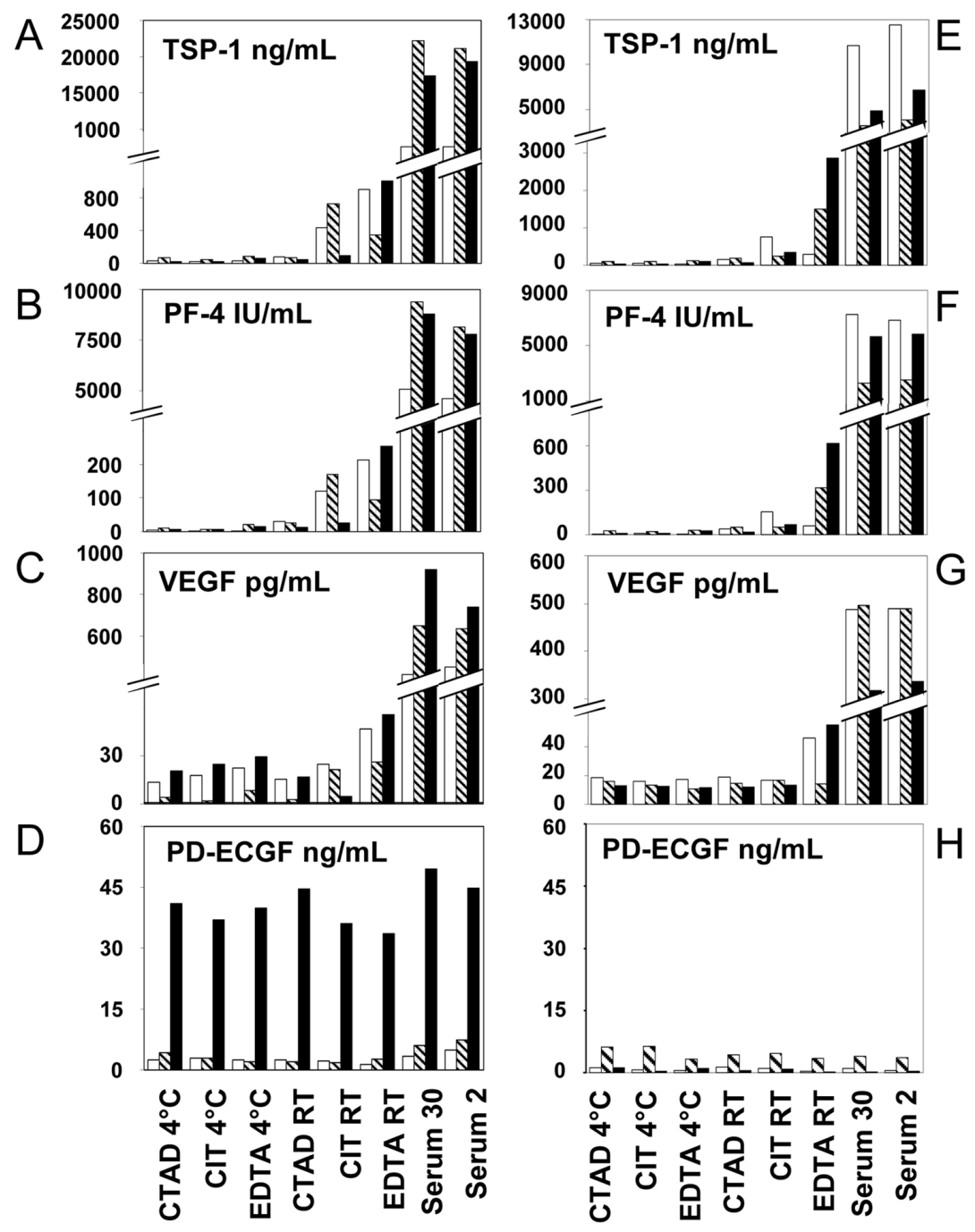

Fig. 1. Blood measurements of angiogenesis factors stored in platelet $\alpha$-granules vary with the method of blood processing. Blood levels of TSP-1 (A, E), PF-4 (B, F), VEGF (C, G) and PD-ECGF (D, H) were determined in three healthy individuals (A-D) and in three pancreatic cancer patients (E-H). Plasma was prepared with CTAD, CIT (citrate) or EDTA at $4^{\circ} \mathrm{C}$ or room temperature (RT). Additionally, serum samples were collected after $30 \mathrm{~min}$ and 2 hours of blood clotting. Parameter concentrations were determined by ELISA. Results obtained for the three healthy individuals or three cancer patients are illustrated separately by white, black and hatched bars.

( 1 female, 2 male). Among the CTAD, citrate and EDTA preparations of healthy blood at $4{ }^{\circ} \mathrm{C}$ no substantial differences in measured concentrations of angiogenesis factors were recorded. At room temperature, plasma processing with the anticoagulants citrate or EDTA resulted in a pronounced rise of detectable TSP-1 and PF-4 levels, while VEGF measurements increased moderately (Fig. 1A-D). In serum preparations all three proteins showed a notable elevation. Mean parameter levels measured in serum as compared to CTAD $4{ }^{\circ} \mathrm{C}$ plasma were 920-fold increased for PF-4, 380-fold for TSP-1 and 75-fold for VEGF. While these angiogenesis factors showed a highly significant correlation of detected parameter levels with blood processing variants $(\mathrm{P} \leqslant 0.001)$, no substantial fluctuations were recorded for PD-ECGF concentrations established in serum or plasma preparations. Unexpectedly, the blood level of PD-ECGF showed a high interindividual variation within the healthy population with no apparent diseaserelated cause.

The impact of blood processing on the measurements of angiogenesis factors in cancer patients (Fig. 1E$\mathrm{H})$ was entirely comparable to the results obtained for healthy individuals. 

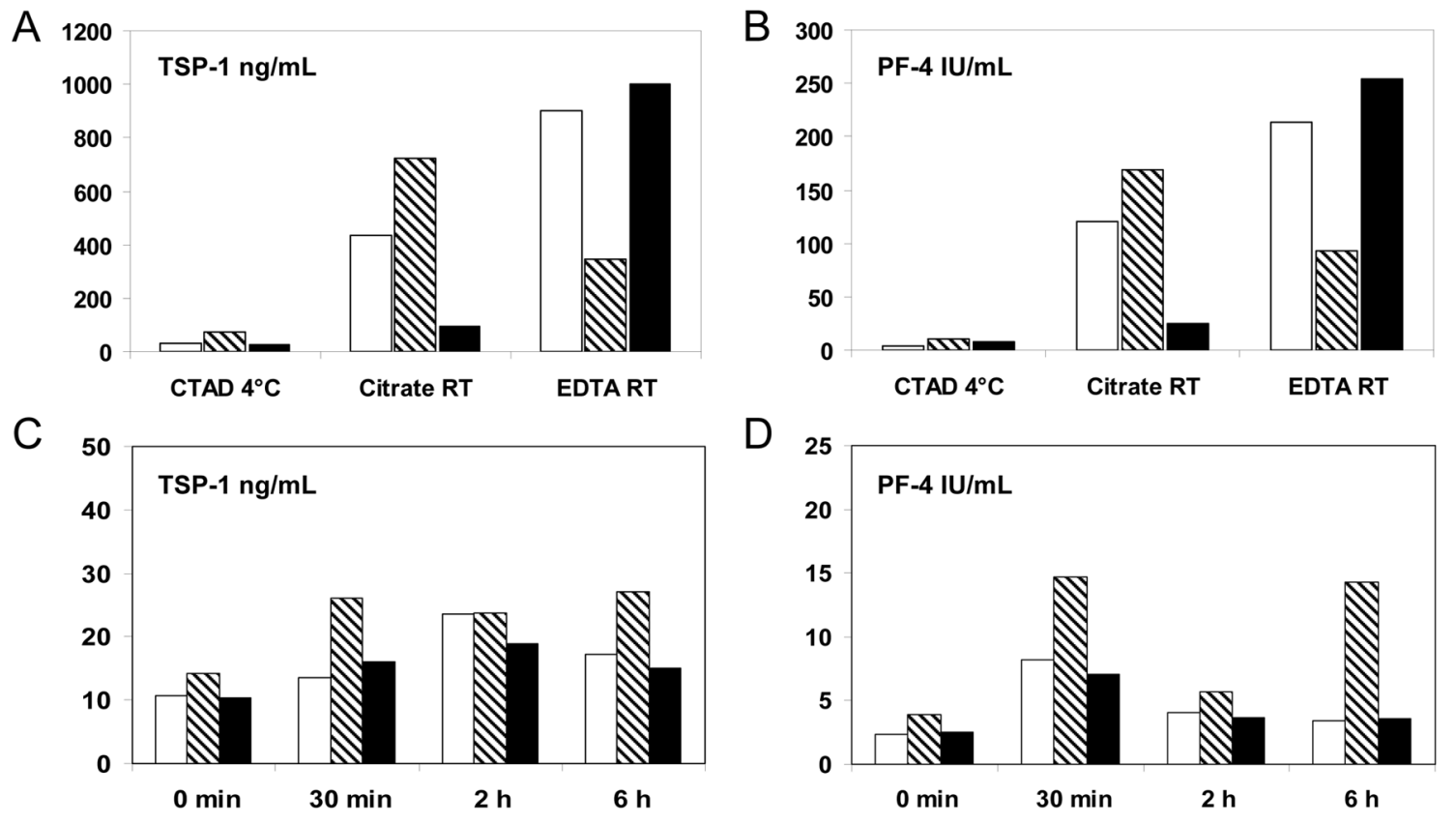

Fig. 2. In vitro fluctuations of angiogenesis parameters show varying magnitude. Concentrations of TSP-1 (A) and PF-4 (B) as determined by ELISA for samples with optimized plasma processing (CTAD at $4^{\circ} \mathrm{C}$ ) within $30 \mathrm{~min}$ of blood withdrawal were compared to levels recorded for suboptimal plasma preparation with citrate or EDTA at room temperature (RT). Furthermore, concentrations of TSP-1 (C) and PF-4 (D) were established by optimized plasma processing (CTAD at $4^{\circ} \mathrm{C}$ ) immediately following blood withdrawal or after blood storage at $4{ }^{\circ} \mathrm{C}$ for 30 min, $2 \mathrm{~h}$ or $6 \mathrm{~h}$. Values obtained for 3 healthy individuals are given and are illustrated separately by white, black and hatched bars.

\subsection{In vitro fluctuations of angiogenesis parameters are erratic}

Having established that the choice of plasma preparation method may lead to an artificial increase of detectable angiogenesis factors (presumably due to partial platelet activation and degranulation), we addressed the question whether the in vitro artifact was due to a reproducible process resulting in a consistent degree of sample "contamination". Therefore, plasma values of TSP-1 and PF-4 obtained with optimized blood processing (with CTAD at $4^{\circ} \mathrm{C}$ ) were directly compared to plasma levels established for citrate or EDTA preparations at room temperature for the three individuals (Fig. 2A and 2B show regrouped data from Fig. 1). The extent of contamination differed substantially among the three individuals and was incoherent for citrate and EDTA preparations indicating an inconsistent process of varying magnitude. However, TSP-1 and PF-4 levels showed a comparable pattern of fluctuation (coregulation).

When evaluating whether the time between blood withdrawal and blood processing would increase the risk of inadvertent platelet activation, we found that storage times between $30 \mathrm{~min}$ to $6 \mathrm{~h}$ resulted in oc- casionally elevated levels of TSP-1 and PF-4 (Fig. 2C and 2D) but no progressive, time-dependent rise in the determined plasma concentration. Of note, the fluctuations of TSP-1 and PF-4 measurements introduced by blood storage were comparably minor, i.e., 10-20 fold lower than the range of "contamination" reached by blood processing with citrate or EDTA at room temperature. CTAD plasma analysis of VEGF and PD-ECGF was entirely unaffected by the extent of blood storage (data not shown).

\subsection{The blood collection procedure affects plasma measurements of platelet-stored angiogenic factors}

In a clinical setting the most frequently used collection systems to draw blood from patients are venflon (18 G needle) and butterfly ( $21 \mathrm{G}$ needle). To evaluate whether differences in blood collection could also affect plasma measurements of platelet-stored angiogenic factors, blood of 10 healthy volunteers was collected with both systems and subjected to optimized plasma preparation (with CTAD at $4{ }^{\circ} \mathrm{C}$ ). Subsequently, samples of 10 volunteers were analyzed for VEGF, TSP1 and PD-ECGF content, and PF-4 plasma values were established for 8 individuals. 

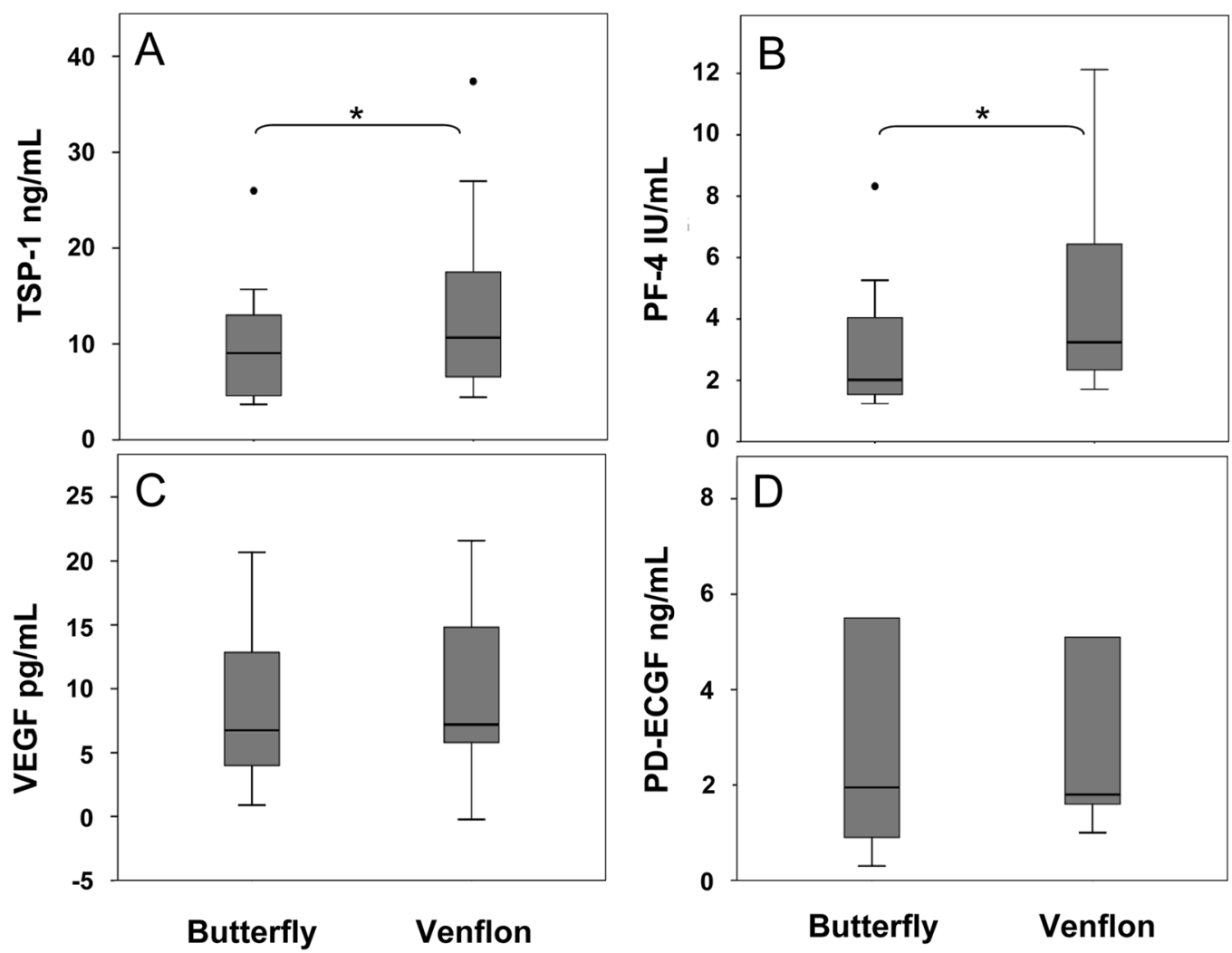

Fig. 3. The choice of blood collection system affects the detection of circulating angiogenesis factors. Plasma values were measured by ELISA for TSP-1 (A), PF-4 (B), VEGF (C) and PD-ECGF (D) after blood withdrawal by venflon or butterfly collection system in 10 healthy individuals. Plasma was prepared with CTAD at $4{ }^{\circ} \mathrm{C}$. Significant differences between groups $(\mathrm{P}<0.05)$ are indicated by asterisk $\left({ }^{*}\right)$.

TSP-1 and PF-4 plasma levels were significantly higher when blood had been drawn using the venflon as compared to the butterfly collection system $(\mathrm{P}=$ 0.028 and $\mathrm{P}=0.012$, respectively). In contrast, plasma values of VEGF and PD-ECGF were not substantially affected by the mode of blood withdrawal (Fig. 3).

\subsection{Inadvertent hemolysis during blood withdrawal impairs plasma measurements of platelet-stored angiogenesis factors}

As accidental hemolysis occurs in clinical routine blood withdrawal (in particular when collecting blood from elderly or cancer patients under therapy), we were interested to assess a possible impact of hemolysis on plasma measurements of platelet-stored angiogenic factors. Thus, 49 plasma samples retrieved from 19 pancreatic cancer patients during therapy were prepared with CTAD at $4^{\circ} \mathrm{C}$ and were analyzed for VEGF, TSP-1, PF-4 and PD-ECGF content. Five of the 49 samples $(10 \%)$ showed signs of hemolysis, i.e. were red tinged. Plasma values of TSP-1 and PF-4 recorded for hemolytic samples were remarkably elevated and significantly higher than for non-hemolysed samples $(\mathrm{P}=0.001$ and $\mathrm{P}=0.002$, respectively). VEGF and PD-ECGF concentrations did not differ significantly between groups (Fig. 4).

\subsection{Optimized blood processing reveals a selective increase of VEGF and TSP-1 (as opposed to $P F-4$ and $P D-E C G F$ ) in pancreatic cancer patients}

Having identified potential pitfalls of determining angiogenesis parameters in blood, we questioned whether previously reported elevated levels of TSP-1, VEGF and PF-4 in cancer patients could be confirmed or disputed when applying optimized blood collection and processing procedures. Hence, 13 locally advanced pancreatic cancer patients prior to chemotherapy were compared to an age-matched control collective of 13 

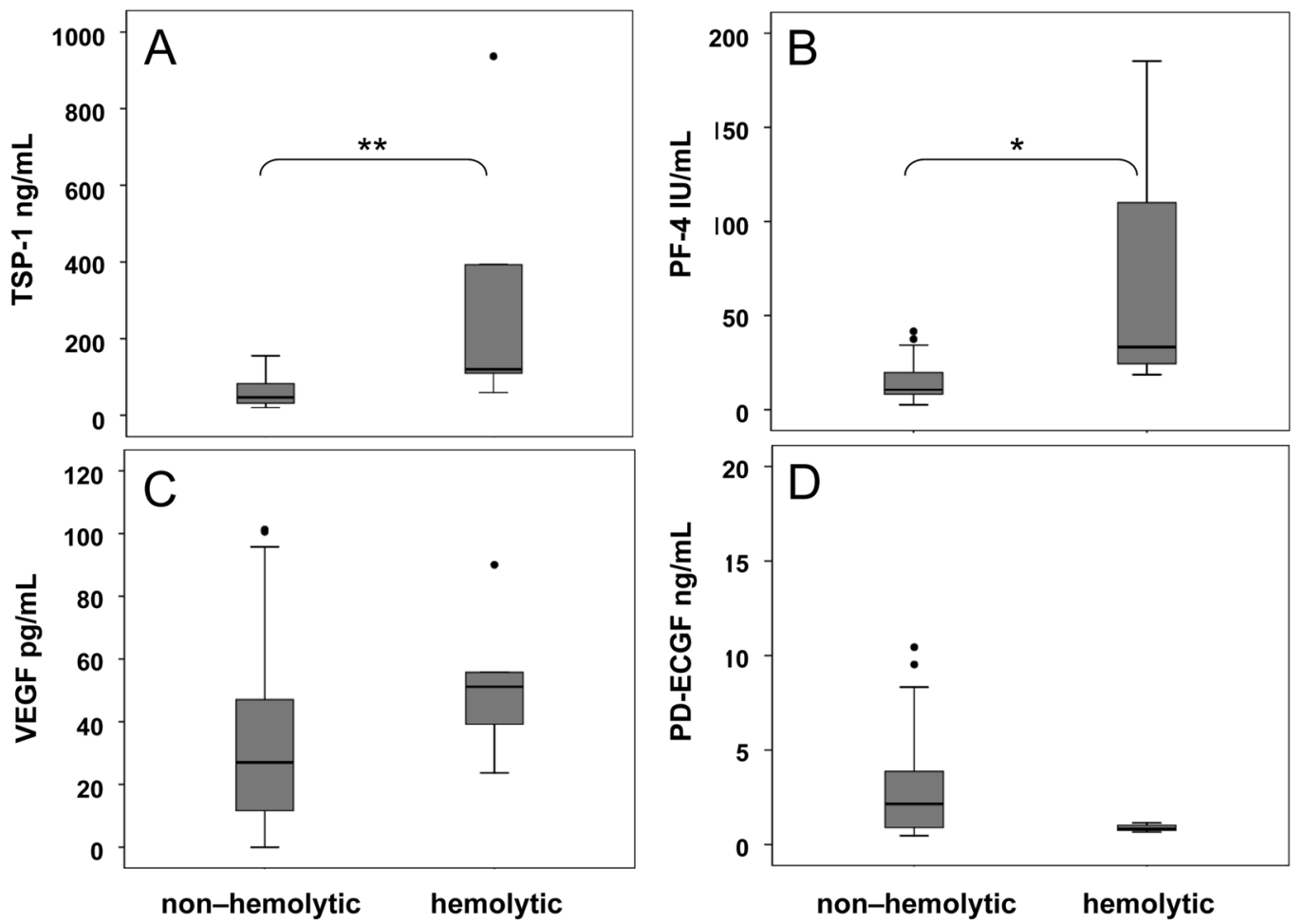

Fig. 4. Hemolysis during blood withdrawal affects the detection of circulating angiogenesis factors. TSP-1 (A), PF-4 (B), VEGF (C) and PD-ECGF (D) plasma values were determined by ELISA in 5 hemolytic and 44 non-hemolytic samples retrieved from pancreatic cancer patients during chemotherapy. Plasma was prepared with CTAD at $4{ }^{\circ} \mathrm{C}$. Significant $(\mathrm{P}<0.05)$ and highly significant $(\mathrm{P} \leqslant 0.001)$ differences between groups are indicated by one $(*)$ or two $(* *)$ asterisks, respectively.

healthy individuals with respect to circulating levels of angiogenesis factors (Fig. 5).

Blood concentrations of TSP-1 and VEGF were found to be significantly elevated in the group of cancer patients (median TSP-1 healthy: $34.5 \mathrm{ng} / \mathrm{mL}$, median TSP-1 cancer: $70.2 \mathrm{ng} / \mathrm{mL}, \mathrm{P}=0.006$; median VEGF healthy: $2.52 \mathrm{pg} / \mathrm{mL}$, median VEGF cancer: $18.24 \mathrm{pg} / \mathrm{mL}, \mathrm{P}<0.001)$. In contrast, plasma levels of PF-4 and PD-ECGF were comparable between healthy individuals and cancer patients (median PF-4 healthy: 13.2 IU/mL, median PF-4 cancer: $13.9 \mathrm{IU} / \mathrm{mL}, \mathrm{P}=$ 0.762; median PD-ECGF healthy: $2.22 \mathrm{ng} / \mathrm{mL}$, median PD-ECGF cancer: $3.36 \mathrm{ng} / \mathrm{mL}, \mathrm{P}=0.742$ ).

\subsection{Monitoring of angiogenesis parameters is commonly conducted with suboptimal procedures}

A literature search for research articles published within the last 2 years was performed as summarized in Table 1. Studies on VEGF, TSP-1, PF-4 and PDECGF were screened for the anticoagulants applied in plasma analysis. We found that the majority of studies used citrate or EDTA rather than CTAD and gave no information on blood processing temperature suggesting that suboptimal procedures of plasma preparation are commonly applied when monitoring blood levels of angiogenesis parameters.

\section{Discussion}

The analysis of angiogenesis factors has become a powerful tool to understand physiological as well as pathological processes of neovascularization. A shift in the well balanced system of pro- and anti-angiogenic factors is associated with various diseases and is known to critically influence disease progression [28,29]. As platelets store and actively sequester angiogenesis factors, they are increasingly recognized to play a major role in the regulation of neovascularization [30].

With respect to plasma measurements of angiogenesis factors, platelets are easily activated during blood processing and may release their content resulting in increased parameter levels. Analysis of platelet-poor/free 
Table 1

Anticoagulants applied in blood analyses of angiogenesis factors as reported for 2008 $2010^{*}$

\begin{tabular}{lccccccc}
\hline Factor & Total & Relevant & Accessible & CTAD & Citrate & EDTA & NA \\
\hline VEGF & $530(100)$ & 36 & 24 & 1 & 4 & 9 & 10 \\
TSP-1 & 30 & 12 & 8 & 1 & 2 & 2 & 3 \\
PF-4 & 34 & 17 & 12 & 3 & 8 & 1 & 0 \\
PD-ECGF & 41 & 7 & 7 & 1 & 0 & 2 & 4 \\
\hline
\end{tabular}

* The total of articles retrieved by PubMed search for the respective angiogenesis factor combined with the term "plasma" was screened for relevant studies. Accessible articles were evaluated for the anticoagulant (CTAD, citrate, EDTA) applied in blood processing or are listed as NA when no information was given. With respect to studies on VEGF, 100 from 530 retrieved articles were analyzed.
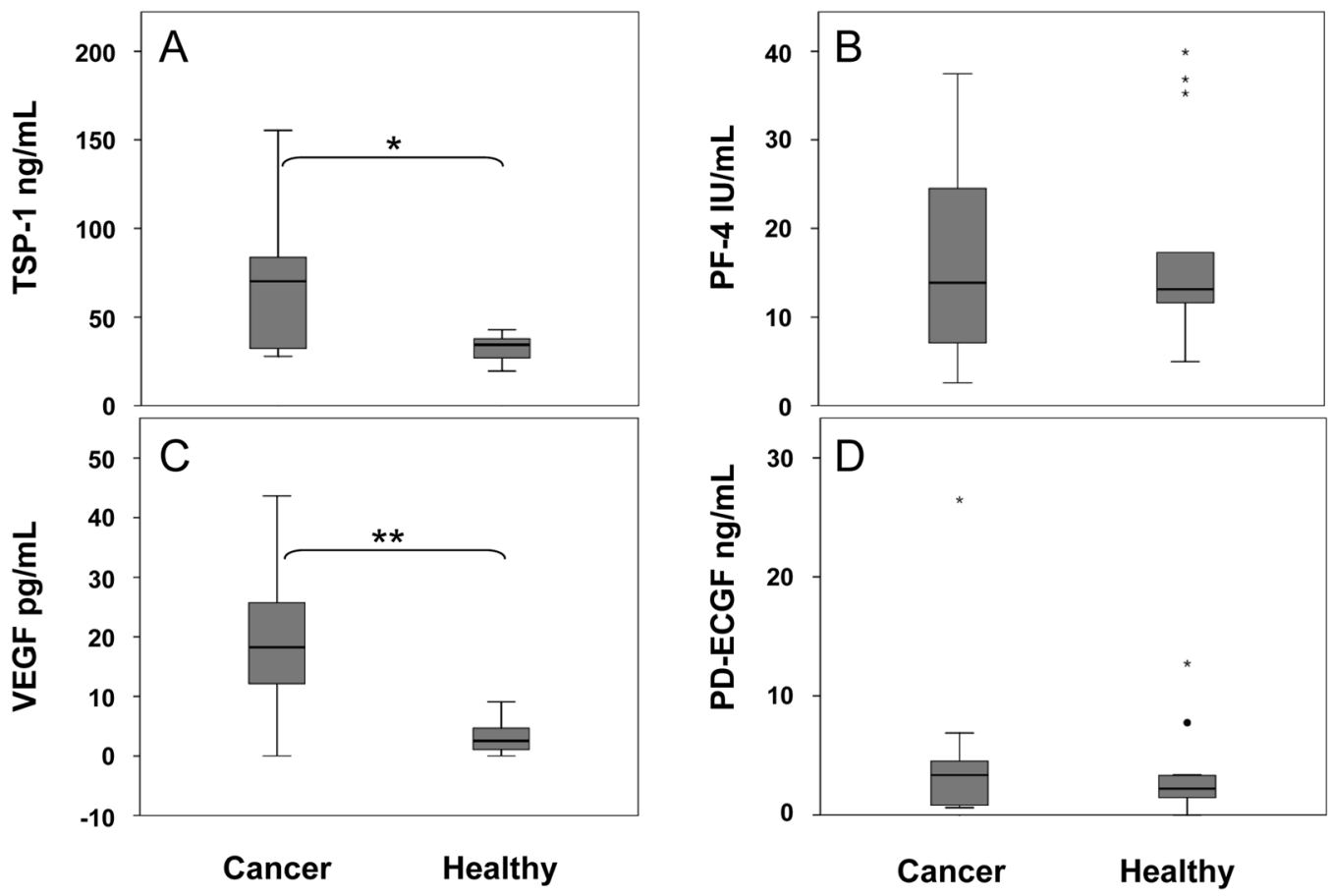

Fig. 5. Pancreatic cancer patients show elevated plasma levels of VEGF and TSP-1. Blood samples of 13 pancreatic cancer patients obtained before therapy were compared to samples from 13 age-matched healthy controls. TSP-1 (A), PF-4 (B), VEGF (C) and PD-ECGF (D) concentrations were determined by ELISA following plasma preparation with CTAD at $4{ }^{\circ} \mathrm{C}$. Significant $(\mathrm{P}<0.05)$ and highly significant $(\mathrm{P} \leqslant 0.001)$ differences between groups are indicated by one $(*)$ or two $(* *)$ asterisks, respectively.

plasma has thus been suggested to determine angiogenesis factors in circulation [21]. However, platelet activation may also occur during the preparation of platelet-poor plasma [22]. The choice of anticoagulant is of importance to avoid pre-analytical variability. In 1999 Wynendaele et al. were first to propose the CTAD mix of four anticoagulants to effectively block platelet activation during plasma preparation for the reliable assessment of circulating VEGF [21]. In contrast to VEGF, comparably little attention has been given to optimizing procedures for the blood analysis of TSP-1 or other platelet-stored angiogenesis factors such as PF-4 and PD-ECGF [31,32]. Thus, we set out to evaluate the impact of blood processing variants on the detection of platelet-stored angiogenesis factors in a more generalized manner. We found that preparation of plateletpoor plasma with $\mathrm{CTAD}$ at $4^{\circ} \mathrm{C}$ yielded reproducible, low values for circulating VEGF, TSP-1, PF4 and PDECGF and did not seem prone to platelet activation upon temperature fluctuation. In contrast, plasma preparations at room temperature using citrate or EDTA were associated with considerable parameter fluctuations of apparently random magnitude suggesting uncontrolled and partial platelet activation during blood processing. 
Of interest, although exposure to low temperatures is commonly avoided in thrombocyte isolation to prevent perturbation of platelet skeletons, the processing at $4^{\circ} \mathrm{C}$ proved preferable in our study possibly related to a reduced risk of platelet rupture during high-speed centrifugation. The application of butterfly $(21 \mathrm{G}$ needle) as opposed to venflon (18 $\mathrm{G}$ needle) blood collection systems, the immediate blood processing after withdrawal, and the exclusion of hemolyzed samples were further shown to avoid pre-analytical variability. Elevated levels of TSP-1 in "suboptimal" plasma preparations generally correlated with high concentrations of PF-4, whereas the increase in VEGF was less pronounced. This is of particular interest as the optimization of blood processing has largely been neglected for TSP1 and PF-4 analyses while these parameters proved to be highly sensitive to in vitro artifacts in our study. Of interest, PD-ECGF measurements were not affected by the choice of anticoagulant or processing temperature. In contrast to the other angiogenesis factors, PDECGF is present in the cytoplasm of thrombocytes and is not stored in $\alpha$-granules. This observation further supports the notion that partial platelet activation during plasma preparation results in platelet degranulation and the selective release of angiogenesis factors stored in platelet granules [33].

Generation of serum is based on the complete activation of platelets in vitro. Hence, serum measurements of platelet-stored angiogenesis factors largely reflect total platelet content [25,34]. As platelets actively participate in the angiogenic process and scavenge tumor-derived VEGF, cancer patients were shown to exhibit increased serum VEGF values and serum analysis was found suitable for evaluating VEGF levels in disease [35]. Concurrent with the early literature, Wiesner et al. recently reported that platelets of metastatic cancer patients exhibit increased values of VEGF [36]. Interestingly, they also detected a higher percentage of activated thrombocytes and a diminished concentration of TSP-1 stored in the platelets of cancer patients as compared to healthy individuals. Thus, in line with the enhanced platelet activation (degranulation) in tumor patients, the granular TSP-1 pool seems to be drained and - in contrast to VEGF - may not be replenished by scavenging of plasma TSP-1. Correspondingly, Gonzalez et al. reported a reduced TSP-1 level in serum as opposed to a higher TSP-1 concentration in plasma of cancer patients when compared to healthy volunteers [37]. These observations illustrate that the concomitant evaluation of serum and plasma values (compared with platelet counts) may yield the most comprehensive picture of the regulation and availability of angiogenesis factors in cancer patients. Optimized plasma preparation without artificial platelet activation is thus of eminent importance to be able to distinguish between circulating and platelet-stored angiogenesis factors, and suboptimal procedures may explain why several studies failed to observe an increased TSP-1 plasma value in cancer patients [38].

Comparably, divergent results concerning the prognostic potential of circulating VEGF and TSP1 have been reported for defined tumor entities and may relate to the mode of blood processing applied $[8,39,40]$. To assess the variability of plasma processing methods in reported studies and evaluate the potential affliction by in vitro platelet activation, we conducted a screen of research articles published within the last 2 years. Despite the fact that optimized protocols for the assessment of VEGF plasma levels have been released more than a decade ago, we found that monitoring of circulating angiogenesis factors was commonly conducted with procedures known to suffer from partial in vitro activation of platelets. This is particularly noteworthy as blood concentrations of platelet-stored angiogenesis factors are increasingly being evaluated for their prognostic and predictive potential in the context of anti-angiogenic cancer therapies. With our comprehensive analysis we thus aim to emphasize the general importance of optimized plasma processing and alert the community to further pitfalls such as the choice of blood collection system, blood storage and accidental hemolysis when determining circulating levels of platelet-stored angiogenesis factors.

\section{Acknowledgments}

This work was partly supported by the Austrian National Bank ("OeNB Jubiläumsfonds" grant no. 12072) and the Medical Scientific Fund of the Mayor of the City of Vienna (grant no. 08064).

\section{References}

[1] M.L. Iruela-Arispe and H.F. Dvorak, Angiogenesis: a dynamic balance of stimulators and inhibitors, Thromb Haemost 78 (1997), 672-677.

[2] D. Hanahan and J. Folkman, Patterns and emerging mechanisms of the angiogenic switch during tumorigenesis, Cell 86 (1996), 353-364.

[3] N. Ferrara, Vascular endothelial growth factor, Arterioscler Thromb Vasc Biol 29 (2009), 789-791. 
[4] R.E. Banks, M.A. Forbes, S.E. Kinsey, A. Stanley, E. Ingham, C. Walters and P.J. Selby, Release of the angiogenic cytokine vascular endothelial growth factor (VEGF) from platelets: significance for VEGF measurements and cancer biology, $\mathrm{Br} J$ Cancer 77 (1998), 956-964.

[5] N. Ferrara, VEGF as a therapeutic target in cancer, Oncology 69(Suppl 3) (2005), 11-16.

[6] J. Adams, P.J. Carder, S. Downey, M.A. Forbes, K. MacLennan, V. Allgar, S. Kaufman, S. Hallam, R. Bicknell, J.J. Walker, F. Cairnduff, P.J. Selby, T.J. Perren, M. Lansdown and R.E. Banks, Vascular endothelial growth factor (VEGF) in breast cancer: comparison of plasma, serum, and tissue VEGF and microvessel density and effects of tamoxifen, Cancer Res $\mathbf{6 0}$ (2000), 2898-2905.

[7] A.O. Kaseb, A. Hanbali, M. Cotant, M.M. Hassan, I. Wollner and P.A. Philip, Vascular endothelial growth factor in the management of hepatocellular carcinoma: a review of literature, Cancer 115 (2009), 4895-4906.

[8] N. Murukesh, C. Dive and G.C. Jayson, Biomarkers of angiogenesis and their role in the development of VEGF inhibitors, Br J Cancer 102 (2010), 8-18.

[9] J. Dawes, K.J. Clemetson, G.O. Gogstad, J. McGregor, P. Clezardin, C.V. Prowse and D.S. Pepper, A radioimmunoassay for thrombospondin, used in a comparative study of thrombospondin, beta-thromboglobulin and platelet factor 4 in healthy volunteers, Thromb Res 29 (1983), 569-581.

[10] L.L. Leung and R.L. Nachman, Complex formation of platelet thrombospondin with fibrinogen, J Clin Invest 70 (1982), 542549 .

[11] L.L. Leung, Role of thrombospondin in platelet aggregation, J Clin Invest 74 (1984), 1764-1772.

[12] M.A. Kowalska, L. Rauova and M. Poncz, Role of the platelet chemokine platelet factor 4 (PF4) in hemostasis and thrombosis, Thromb Res 125 (2010), 292-296.

[13] R.J. Sharpe, H.R. Byers, C.F. Scott, S.I. Bauer and T.E. Maione, Growth inhibition of murine melanoma and human colon carcinoma by recombinant human platelet factor $4, J$ Natl Cancer Inst 82 (1990), 848-853.

[14] S. Kazerounian, K.O. Yee and J. Lawler, Thrombospondins in cancer, Cell Mol Life Sci 65 (2008), 700-712.

[15] A. Zaslavsky, K.H. Baek, R.C. Lynch, S. Short, J. Grillo, J. Folkman, J.E. Italiano, Jr. and S. Ryeom, Platelet-derived thrombospondin- 1 is a critical negative regulator and potential biomarker of angiogenesis, Blood 115 (2010), 4605-4613.

[16] R. Yabkowitz, P.J. Mansfield, V.M. Dixit and S.J. Suchard, Motility of human carcinoma cells in response to thrombospondin: relationship to metastatic potential and thrombospondin structural domains, Cancer Res 53 (1993), 378387.

[17] V. Abbasciano, D. Tassinari, S. Sartori, L. Trevisani, D. Arcudi, M.P. Bianchi and A. Liboni, Usefulness of coagulation markers in staging of gastric cancer, Cancer Detect Prev 19 (1995), 331-336

[18] V. Dymicka-Piekarska, A. Butkiewicz, H. Kemona, M. Gryko and M. Mantur, Does colorectal cancer clinical advancement and surgical treatment affect platelet factor 4 concentration, Pol Merkur Lekarski 17 (2004), 575-578.

[19] T. Shaw, R.H. Smillie and D.G. MacPhee, The role of blood platelets in nucleoside metabolism: assay, cellular location and significance of thymidine phosphorylase in human blood, Mutat Res 200 (1988), 99-116.

[20] J. Fujimoto, H. Sakaguchi, I. Aoki and T. Tamaya, The value of platelet-derived endothelial cell growth factor as a novel predictor of advancement of uterine cervical cancers, Cancer Res 60 (2000), 3662-3665.

[21] W. Wynendaele, R. Derua, M.F. Hoylaerts, A. Pawinski, E. Waelkens, E.A. de Bruijn, R. Paridaens, W. Merlevede and A.T. van Oosterom, Vascular endothelial growth factor measured in platelet poor plasma allows optimal separation between cancer patients and volunteers: a key to study an angiogenic marker in vivo? Ann Oncol 10 (1999), 965-971.

[22] R. Dittadi, S. Meo, F. Fabris, G. Gasparini, D. Contri, M. Medici and M. Gion, Validation of blood collection procedures for the determination of circulating vascular endothelial growth factor (VEGF) in different blood compartments, Int J Biol Markers 16 (2001), 87-96.

[23] M.L. George, S.A. Eccles, M.G. Tutton, A.M. Abulafi and R.I. Swift, Correlation of plasma and serum vascular endothelial growth factor levels with platelet count in colorectal cancer: clinical evidence of platelet scavenging? Clin Cancer Res 6 (2000), 3147-3152.

[24] E. Gunsilius, A. Petzer, G. Stockhammer, W. Nussbaumer, P. Schumacher, J. Clausen and G. Gastl, Thrombocytes are the major source for soluble vascular endothelial growth factor in peripheral blood, Oncology 58 (2000), 169-174.

[25] H.M. Verheul, K. Hoekman, S. Luykx-de Bakker, C.A. Eekman, C.C. Folman, H.J. Broxterman and H.M. Pinedo, Platelet: transporter of vascular endothelial growth factor, Clin Cancer Res 3 (1997), 2187-2190.

[26] C. Brostjan, A. Bayer, A. Zommer, A. Gornikiewicz, S. Roka, T. Benko, R. Yaghubian, R. Jakesz, G. Steger, M. Gnant, J. Friedl and A. Stift, Monitoring of circulating angiogenic factors in dendritic cell-based cancer immunotherapy, Cancer 98 (2003), 2291-2301.

[27] C. Brostjan, K. Gebhardt, B. Gruenberger, V. Steinrueck, H. Zommer, H. Freudenthaler, S. Roka and T. Gruenberger, Neoadjuvant treatment of colorectal cancer with bevacizum$\mathrm{ab}$ : the perioperative angiogenic balance is sensitive to systemic thrombospondin-1 levels, Clin Cancer Res 14 (2008), 2065-2074.

[28] K. Werther, S. Sorensen, I.J. Christensen and H.J. Nielsen, Circulating vascular endothelial growth factor six months after primary surgery as a prognostic marker in patients with colorectal cancer, Acta Oncol 42 (2003), 837-845.

[29] K. Fujisaki, K. Mitsuyama, A. Toyonaga, K. Matsuo and K. Tanikawa, Circulating vascular endothelial growth factor in patients with colorectal cancer, Am J Gastroenterol 93 (1998), 249-252.

[30] G.L. Klement, T.T. Yip, F. Cassiola, L. Kikuchi, D. Cervi, V. Podust, J.E. Italiano, E. Wheatley, A. Abou-Slaybi, E. Bender, N. Almog, M.W. Kieran and J. Folkman, Platelets actively sequester angiogenesis regulators, Blood 113 (2009), 28352842.

[31] R. Ohkawa, Y. Hirowatari, K. Nakamura, S. Ohkubo, H. Ikeda, M. Okada, M. Tozuka, K. Nakahara and Y. Yatomi, Platelet release of beta-thromboglobulin and platelet factor 4 and serotonin in plasma samples, Clin Biochem 38 (2005), 1023-1026.

[32] G. Bergseth, K.T. Lappegard, V. Videm and T.E. Mollnes, A novel enzyme immunoassay for plasma thrombospondin. Comparison with beta-thromboglobulin as platelet activation marker in vitro and in vivo, Thromb Res 99 (2000), 41-50.

[33] P. Starlinger, H.P. Moll, A. Assinger, C. Nemeth, K. Hoetzenecker, B. Gruenberger, T. Gruenberger, I. Kuehrer, S.F. Schoppmann, M. Gnant and C. Brostjan, Thrombospondin-1: a unique marker to identify in vitro platelet activation when monitoring in vivo processes, J Thromb Haemost 8 (2010), 1809-1819. 
[34] P. Salven, A. Orpana and H. Joensuu, Leukocytes and platelets of patients with cancer contain high levels of vascular endothelial growth factor, Clin Cancer Res 5 (1999), 487-491.

[35] H.M. Verheul and H.M. Pinedo, The importance of platelet counts and their contents in cancer, Clin Cancer Res 9 (2003), 3219-3221.

[36] T. Wiesner, S. Bugl, F. Mayer, J.T. Hartmann and H.G. Kopp, Differential changes in platelet VEGF, Tsp, CXCL12, and CXCL4 in patients with metastatic cancer, Clin Exp Metastasis 27 (2010), 141-149.

[37] F.J. Gonzalez, A. Rueda, I. Sevilla, L. Alonso, V. Villarreal, E. Torres and E. Alba, Shift in the balance between circulating thrombospondin-1 and vascular endothelial growth factor in cancer patients: relationship to platelet alpha-granule content and primary activation, Int J Biol Markers 19 (2004), 221-228.

[38] A.Z. Dudek and H. Mahaseth, Circulating angiogenic cytokines in patients with advanced non-small cell lung cancer: correlation with treatment response and survival, Cancer Invest 23 (2005), 193-200.

[39] K. Werther, I.J. Christensen and H.J. Nielsen, Prognostic impact of matched preoperative plasma and serum VEGF in patients with primary colorectal carcinoma, Br J Cancer $\mathbf{8 6}$ (2002), 417-423.

[40] R.M. Roumen, G.D. Slooter, F.A. Croiset van Uchelen and L.V. Huib, Preoperative serum vascular endothelial growth factor is not a marker for subsequent recurrence during longterm follow-up of colorectal cancer patients, Dis Colon Rectum 48 (2005), 1070-1075. 


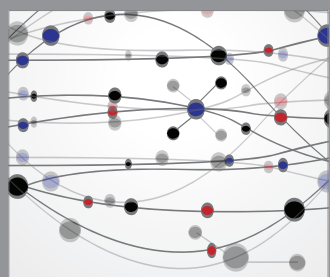

The Scientific World Journal
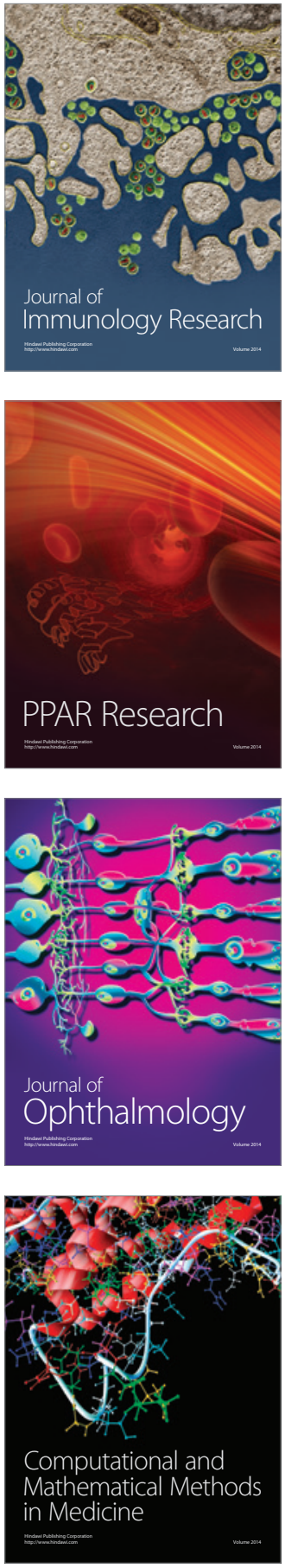

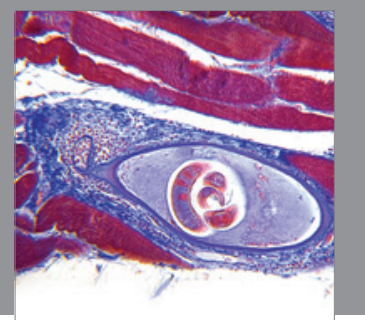

Gastroenterology

Research and Practice
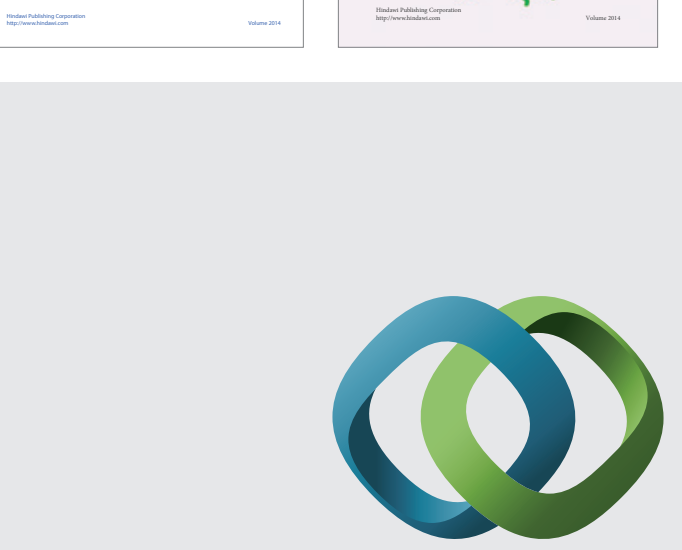

\section{Hindawi}

Submit your manuscripts at

http://www.hindawi.com
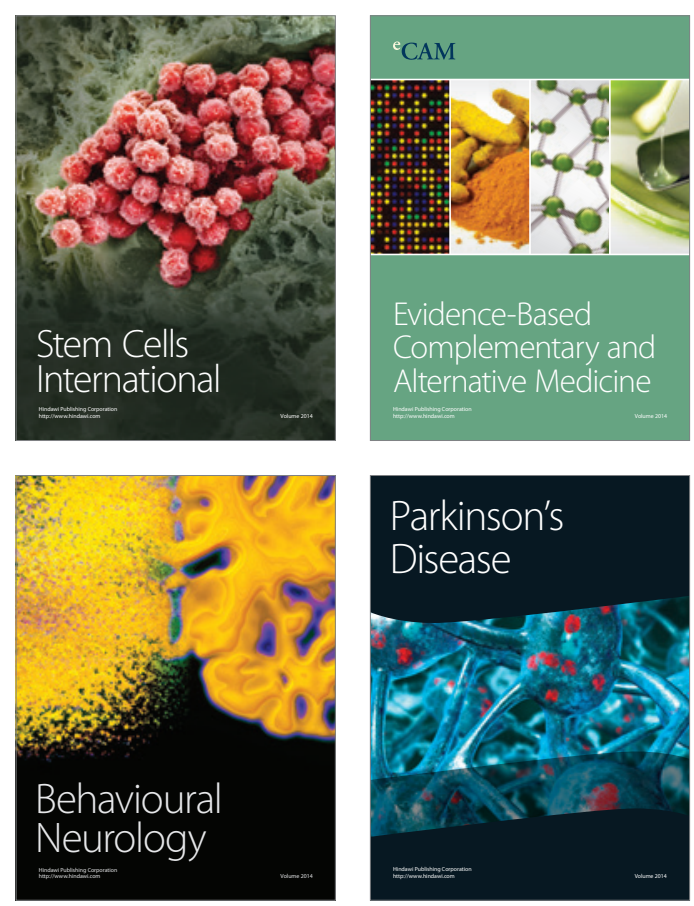

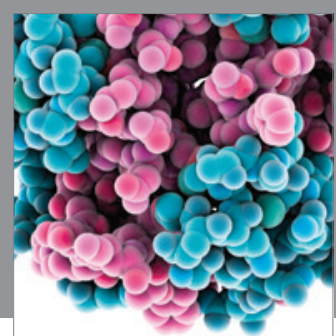

Journal of
Diabetes Research

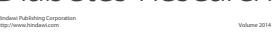

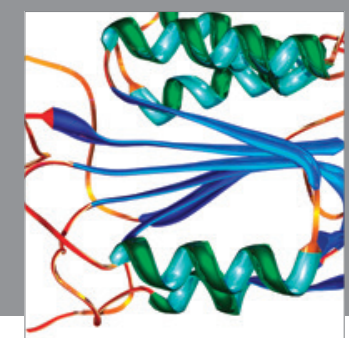

Disease Markers
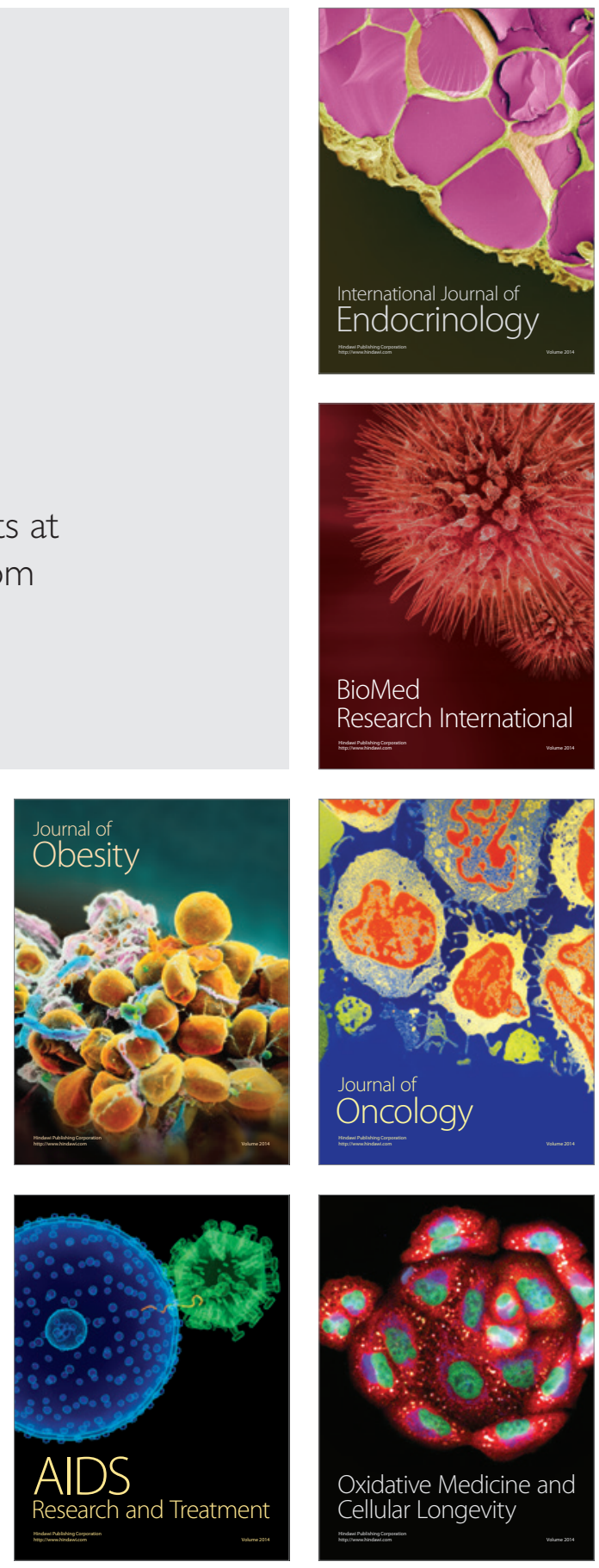